\title{
TOLOSA HUNT SYNDROME: A RARE CASE REPORT
}

\section{S. Sivaranjani1 ${ }^{1}$, M. Loganathan², Harshila Jain ${ }^{3}$, Datta Gulnar Pandian4, Vasudev Anand Rao ${ }^{5}$}

\section{HOW TO CITE THIS ARTICLE:}

S. Sivaranjani, M. Loganathan, Harshila Jain, Datta Gulnar Pandian, Vasudev Anand Rao. "Tolosa Hunt Syndrome: A Rare Case Report". Journal of Evolution of Medical and Dental Sciences 2015; Vol. 4, Issue 18, March 02; Page: 3192-3196, DOI: $10.14260 /$ jemds/2015/463

ABSTRACT: A 39 years old woman presented with painful loss of vision in left eye for 3 days. Patient had similar complaints in left eye 3 months back. On examination, her visual acuity was perception of light with accurate projection of rays with RAPD. Severe ptosis was present in left eye. The patient had restricted extra ocular movement in all gazes and numbness in periorbital region. Right eye examination was unremarkable. The case was diagnosed as multiple cranial nerve palsy and MRI contrast revealed abscess in the left orbital apex region involving, left cavernous sinus, extra axial left temporal lobe, extending into superior orbital fissure and associated patchy meningeal involvement. Tolosa-Hunt syndrome (THS) is a rare disorder characterized by severe unilateral headaches with multiple cranial nerve palsies, usually involving the third, fourth, fifth, and sixth cranial nerves and periorbital pain, along with weakness and paralysis of extra ocular muscles.[1] The exact cause of THS is not known, but the disorder is associated with inflammation of cavernous sinus and superior orbital fissure.

KEYWORDS: Tolosa hunt syndrome, multiple cranial nerve palsy, ptosis.

INTRODUCTION: Treatment of THS is corticosteroids and immunosuppressive agents (such as methotrexate or azathioprine.[2] Patients usually respond to corticosteroids, and spontaneous remission can occur, although extra-ocular muscles may not recover completely.[3] Roughly 30-40\% of patients who are treated for THS experience a relapse.[2] The prognosis of THS is usually considered good.

A 39 years old woman presented with painful loss of vision in left eye for 3 days. Patient had similar complaints in left eye 3 months back. On examination, her visual acuity was perception of light with accurate projection of rays with RAPD. Severe ptosis was present in left eye. The patient had restricted extra ocular movement in all gazes and numbness in periorbital region. Right eye examination was unremarkable. The case was diagnosed as multiple cranial nerve palsy and MRI contrast revealed abscess in the left orbital apex region involving, left cavernous sinus, extra axial left temporal lobe, extending into superior orbital fissure and associated patchy meningeal involvement.

Tolosa-Hunt syndrome (THS) is a rare disorder characterized by severe unilateral headaches with multiple cranial nerve palsies, usually involving the third, fourth, fifth, and sixth cranial nerves and periorbital pain, along with weakness and paralysis of extra ocular muscles.[1] The exact cause of THS is not known, but the disorder is associated with inflammation of cavernous sinus and superior orbital fissure.

Treatment of THS is corticosteroids and immunosuppressive agents (such as methotrexate or azathioprine).[2] Patients usually respond to corticosteroids, and spontaneous remission can occur, although extra-ocular muscles may not recover completely. ${ }^{[3]}$ Roughly $30-40 \%$ of patients who are treated for THS experience a relapse. [2] The prognosis of THS is usually considered good. 


\section{CASE REPORT}

We report a rare case of Tolosa-Hunt syndrome with recurrence of multiple cranial nerve palsy and its response to steroids.

CASE REPORT: A 39 years old woman presented with painful loss of vision in left eye for 3 days. On examination, her visual acuity was perception of light with accurate projection of rays and RAPD. Severe ptosis was present in left eye. The patient had restricted extra ocular movement in all gazes and numbness in periorbital region. Right eye examination was unremarkable. The case was diagnosed as multiple cranial nerve palsy.

MRI contrast revealed abscess in the left orbital apex region involving, left cavernous sinus, extra axial left temporal lobe, extending into superior orbital fissure and associated patchy meningeal involvement. Complete blood count, Urine routine was normal. VDRL, Mantoux test, HIV serology, CSF Tb PCR negative. Chest X ray PA view was normal.

Oral methyl prednisolone $16 \mathrm{mg}$ thrice a day was started. After two days of steroid treatment, pain in the left eye reduced, ptosis and extra ocular movements improved. After one week, methyl prednisolone dose was tapered. With two weeks of steroid treatment, vision improved to finger counting at half meter with mild ptosis and restriction of extra ocular movements.

During follow up, in four weeks complete recovery of ptosis and extra ocular movements occurred. Vision improved to finger counting at one meter. Repeat MRI was done in four weeks which revealed that inflammation and edema in left cavernous sinus, extra axial left temporal lobe, and superior orbital fissure has reduced.

Patient had similar complaints in left eye 3 months back which improved completely with steroid treatment.

DISCUSSION: Nonspecific inflammation (noncaseating granulomatous or nongranulomatous) within the cavernous sinus or superior orbital fissure is the cause for constant pain, which characterizes the onset of this disorder. Ophthalmoparesis occurs when cranial nerves III, IV, and VI are involved by granulomatous inflammation. Pupillary dysfunction is related to injury to the sympathetic fibers in the cavernous portion of internal carotid artery or parasympathetic fibers that surround the oculomotor nerve. Trigeminal nerve involvement causes paresthesia of the forehead and periorbital area. Pathological involvement beyond the cavernous sinus, superior orbital fissure, or apex of the orbit occurs rarely, and the disorder is part of a continuum with idiopathic orbital pseudo tumor, with which it shares histopathologic features. Spontaneous remissions and relapses may occur in up to $40 \%$ of the patients.

The disorder is rare during the first 2 decades of life; in people older than 20 years, it appears to have an even distribution. When THS occurs in children, the course of the disorder appears to be similar to that experienced by adults.[3] The disorder may threaten sight if untreated and inflammation extends beyond the cavernous sinus to affect the optic nerve. The diagnosis of Tolosa-Hunt syndrome is usually one of exclusion.

MRI [4] of the brain and orbit with and without contrast, magnetic resonance angiography or digital subtraction angiography, and CT scan of the brain and orbit with and without contrast may all be useful. Inflammatory changes in the cavernous sinus, superior orbital fissure, and/or orbital apex are typically observed on high-resolution contrast-enhanced imaging. Corticosteroids are the treatment of choice, usually providing significant pain relief within 24-72 hours of therapy initiation. 
Ophthalmoparesis usually requires weeks to months for resolution; indeed. Ophthalmoparesis may not completely resolve in some cases depending on the degree of inflammation and the aggressiveness of therapy. For refractory cases, azathioprine, methotrexate, or radiation therapy has been employed.[5] Surgical extirpation is generally not a feasible treatment of Tolosa-Hunt syndrome; the primary value of surgical intervention is for histopathologic diagnosis.

If a patient is unable to tolerate steroid therapy, other immunosuppressive therapy may be considered. Typically, the prognosis is considered good. Relapse can occur in as many as $40 \%$ of patients successfully treated for Tolosa-Hunt syndrome. Our patient had recurrence in 3 months after the first episode of ophthalmoplegia. This emphasizes the importance of long term follow up after tapering of steroids.

\section{REFERENCES:}

1. Hunt WE, Meagher JN, Lefever HE, Zerman Z. Painful ophthalmoplegia: its relation to indolent inflammation of the cavernous sinus. Neurology 1961; 11: 56-62.

2. Justine R Smith, James T Rosenbaum. A role for methotrexate in the management of nonInfectious orbital inflammatory disease. Br J Ophthalmol 2001; 85: 1220-1224.

3. Zanus C, Furlan C, Costa P, Cosentini D, Carrozzi M. The Tolosa-Hunt syndrome in children: A case report. Cephalalgia 2009; 29: 1232-1237.

4. Goto Y, Hosokawa S, Goto I, et al. Abnormality in the cavernous sinus in three patients with Tolosa-Hunt syndrome: MRI and CT findings. J. Neurol Neurosurg Psychiatry 1990; 53: 231-4.

5. Cohn DF, Carasso R, Streifler M. Painful ophthalmoplegia: The Tolosa-Hunt syndrome. Eur Neurol. 1979; 18: 373-81.

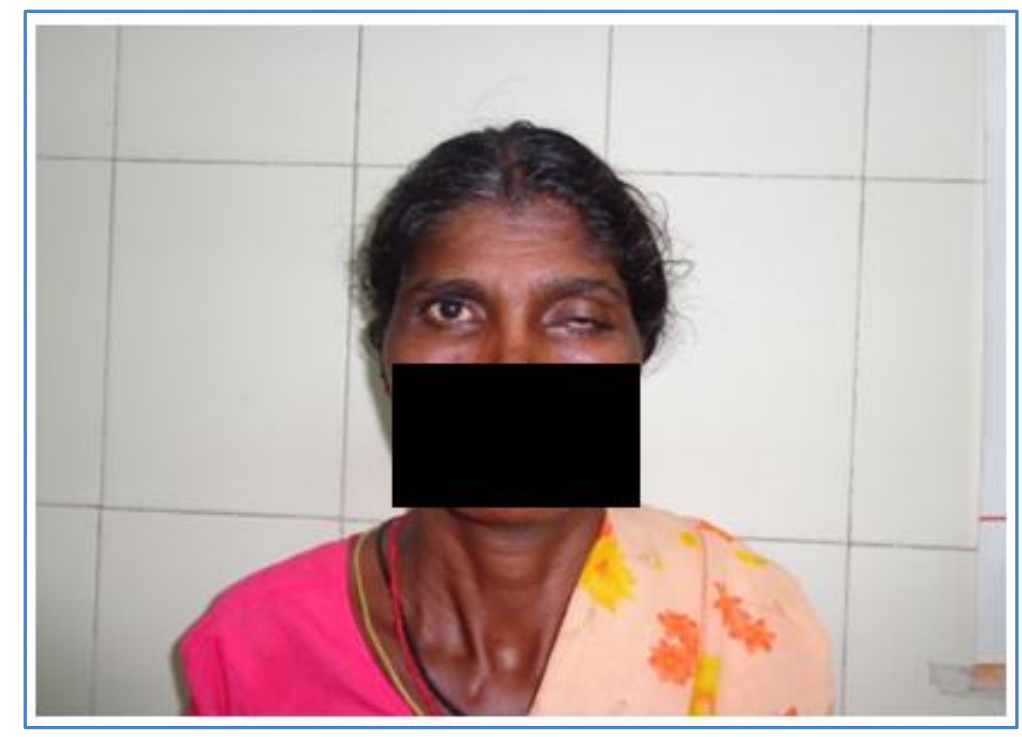

Figure 1: Severe ptosis in primary gaze 


\section{CASE REPORT}

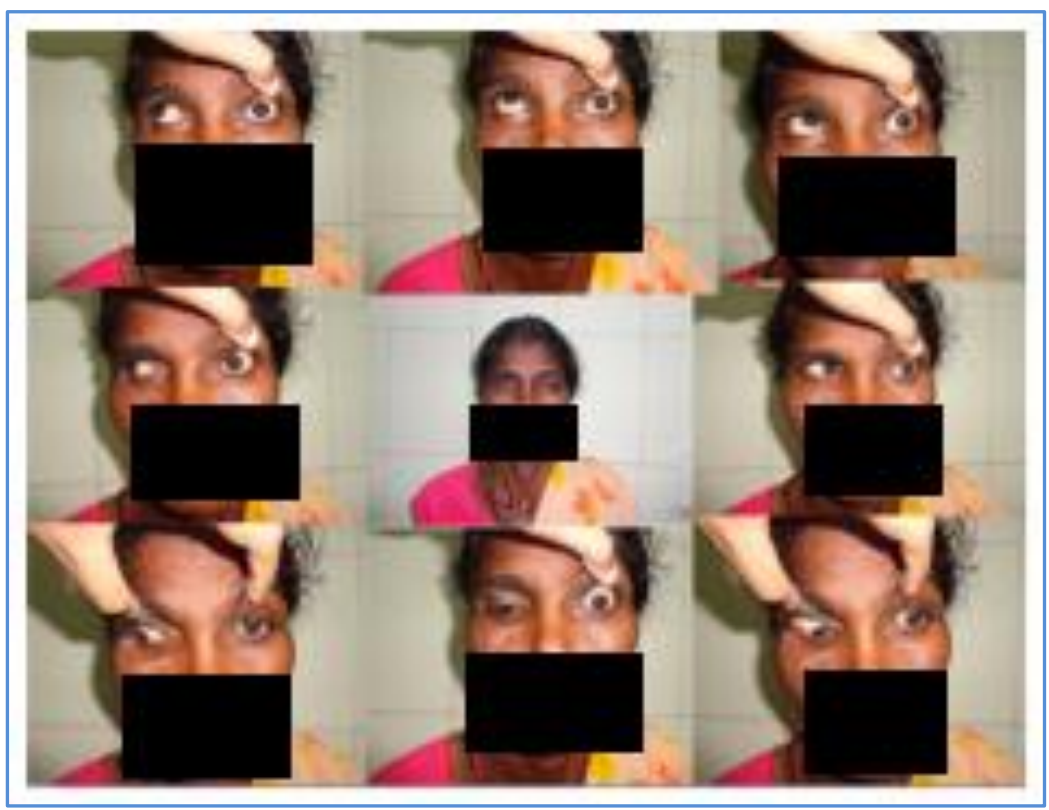

Figure 2: Restricted extra ocular movements in all gazes in LE

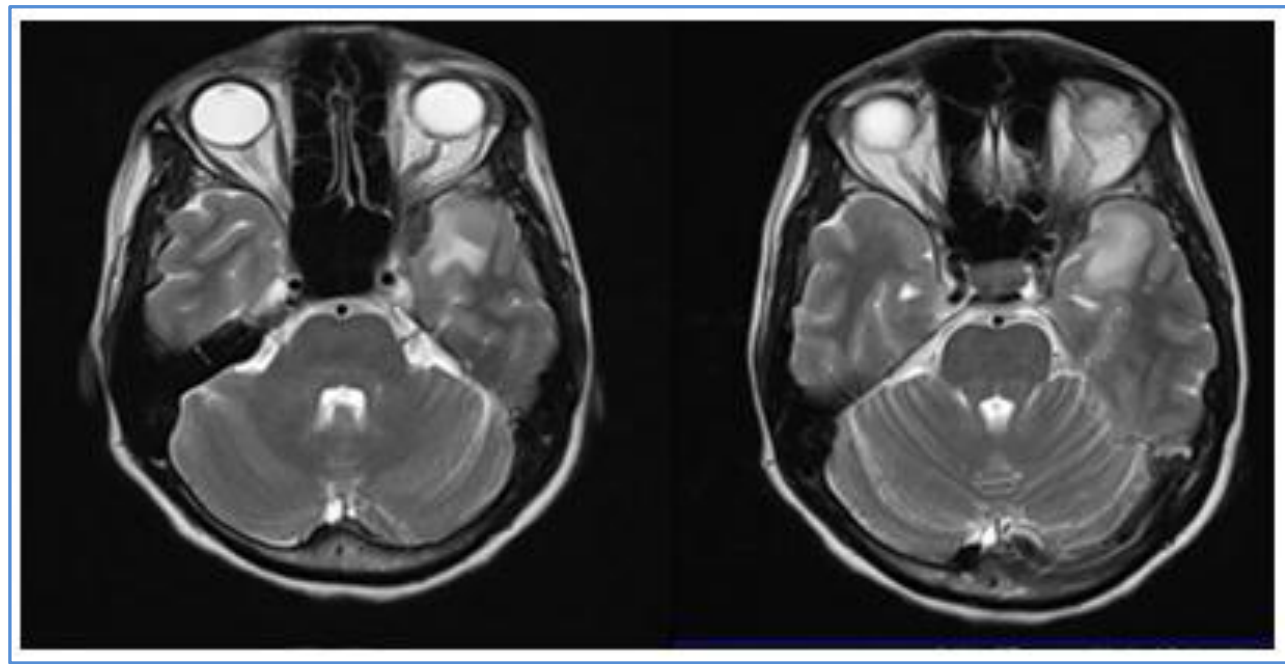

Figure 3: Abscess in the left orbital apex region involving left cavernous sinus, extra axial left temporal lobe, extending into superior orbital fissure and associated patchy meningeal involvement 


\section{AUTHORS:}

1. S. Sivaranjani

2. M. Loganathan

3. Harshila Jain

4. Datta Gulnar Pandian

5. Vasudev Anand Rao

\section{PARTICULARS OF CONTRIBUTORS:}

1. Assistant Professor, Department Ophthalmology, Sri Venkateshwaraa Medical College \& Research Centre.

2. Professor, Department Ophthalmology, Sri Venkateshwaraa Medical College \& Research Centre.

3. Assistant Professor, Department Ophthalmology, Sri Venkateshwaraa Medical College \& Research Centre.

FINANCIAL OR OTHER COMPETING INTERESTS: None
4. Assistant Professor, Department Ophthalmology, Sri Venkateshwaraa Medical College \& Research Centre.

5. Professor \& HOD, Department Ophthalmology, Sri Venkateshwaraa Medical College \& Research Centre.

\section{NAME ADDRESS EMAIL ID OF THE} CORRESPONDING AUTHOR:

Dr. S. Sivaranjani, Assistant Professor, Department Ophthalmology, Sri Venkateshwaraa Medical College \& Research Centre, Ariyur, Puducherry-605102, India.

E-mail: drsivaranjani.s@gmail.com

Date of Submission: 29/01/2015.

Date of Peer Review: 30/01/2015.

Date of Acceptance: 20/02/2015.

Date of Publishing: 02/03/2015. 